\title{
What the educators are saying
}

\author{
Identifying poorly performing \\ junior doctors \\ Being able to identify poorly \\ performing doctors early in their \\ careers is an attractive idea. A recent \\ qualitative study describes the \\ development of a $360^{\circ}$ appraisal tool \\ for use in the intern (preregistration \\ house officer) year. Focus group data \\ identified 51 critical behaviours \\ grouped under eight dimensions: \\ clinical skills, practical skills, patient \\ investigation, patient management, \\ communication, decision making, \\ teamwork, and professionalism. \\ Appraisers can be the educational \\ supervisor, specialist registrars, \\ fellows, senior house officers, and \\ nurses. The tool now needs to be \\ validated.
}

Medical Teacher 2004;26:63-70

Unprofessional behaviour in medical school is associated with disciplinary breaches after graduation

A case-control study (68 cases and 196 matched controls) conducted in one Californian medical school has shown that graduates who behaved unprofessionally in medical school were more likely to be subject to disciplinary action by the state medical board later in their careers. These results emphasise the importance of teaching about professionalism in undergraduate curriculums. They also highlight, yet again, the importance of identifying at undergraduate level any problems that might have an impact on behaviour and performance after graduation.

Academic Medicine 2004;79:244-9

Overcoming negativity about reflective portfolio assessment

Reflective portfolios are an increasingly common assessment method in which students document their course work, learning, progression, and reflections on experience. But studies have indicated that many medical students aren't too keen on them. They see reflection as difficult to understand and hard work, and only to be engaged in if it is compulsory. A recent quantitative survey in a UK medical school found, perhaps unsurprisingly, that undergraduate students with more positive views about reflective portfolios performed better in portfolio based assessment. Overall, students' views about portfolios were neutral. The authors recommend that portfolio based

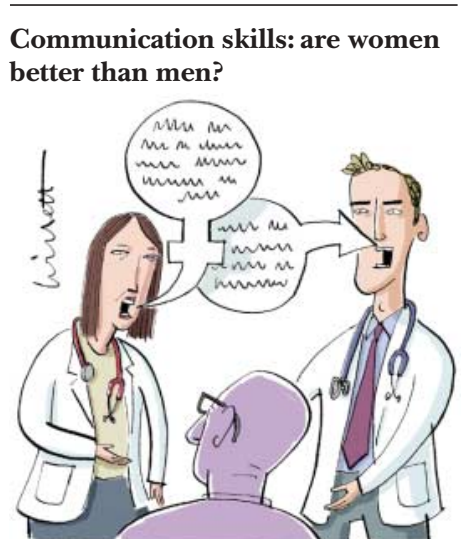

A four year survey (1997-2000) of final year examination results in a UK medical school showed that female students outperformed male students in communication skill OSCE (objective structured clinical examinations) stations. More worryingly, men's performance worsened over the last two years of the study.. The difference between the sexes, though small, suggests that medical educators should pay particular attention to male students'training in communications skills.

Medical Education 2004;38:129-37

assessment should be introduced earlier and that students should receive additional training to build their confidence in using this form of assessment and learning.

Medical Education 2004;38:125-8

Behavioural and social sciences should be integrated into medical school curriculums

Behavioural and social factors, such as smoking, a sedentary lifestyle, and poor diet, are linked to about half of all deaths in the United States. To find out what medical schools are teaching students about the behavioural and social sciences, the US Institute of Medicine looked at curriculum databases. The information available couldn't sufficiently describe curriculum content, teaching techniques, or assessment methodologies, but did result in some recommendations. As well as suggesting the development of a new national behavioural and social sciences database, the report recommends providing medical students with an integrated behavioural and social sciences curriculum throughout the four years at medical school. For this there are six areas of interest: mind-body interactions in health and disease, patient behaviour, physician role and behaviour, physician-patient interactions, social and cultural issues in health care, and health policy and economics. www.iom.edu/report.asp?id $=19413$

Difference in personality types affects doctor-patient communication

Most complaints about doctors relate to poor communication skills, not clinical competence. Personality differences between doctors and their patients may be one of the factors that leads to problems of communication. Using the Myers-Briggs type indicator, Clack et al found that doctors' personality profiles differed significantly from adult norms in the United Kingdom. Medical students and doctors in training should be helped to become aware of their own personality types and how they differ from those of their patients, the authors say. They also recommend incorporating an awareness of apparent differences between doctors' and patients' personalities into programmes for teaching communication and consultation skills.

Medical Education 2004;38:177-86

Admissions OSCE looks promising Interviews are still popular in many admission procedures. Although they have high face value they are not very predictive. The innovative admissions procedure for the medical school at McMaster University is based on the OSCE (objective structured clinical examination). The multi mini-interview, an OSCE-type approach using 10 different short interviews and various raters, was tried out on 117 candidates at McMaster. This approach allows multiple samples of the candidate's abilities, diminishes the influence of chance and bias, and allows for more flexibility in designing stations - and fewer resources are needed than for traditional interviews. The preliminary results look promising but it is too soon yet to know its predictive validity.

\section{Medical Education 2004;38:314-26}

Lambert Schuwirthsenior lecture (L.Schuwirth@educ.unimaas.nl) Department of Educational Development and Research, University of Maastricht, Netherlands Peter Cantillon senior lecturer (peter.cantillon@nuigalway.ie) Department of General Practice, National University of Ireland, Galway, Republic of Ireland 